\title{
Coupling of phonons to a helium atom adsorbed on graphite
}

\author{
R. D. Williams, M. W. Cole, and S. E. Koonin \\ California Institute of Technology, Pasadena, California 91125
}

(Received 22 February 1983)

\begin{abstract}
We compute the self-energy for a ${ }^{4} \mathrm{He}$ atom adsorbed on graphite to second order in the phonon coupling. The phonon contributions amount to several degrees Kelvin. The imaginary part corresponds to a lifetime of some $10^{-11} \mathrm{~s}$.
\end{abstract}

\section{INTRODUCTION}

The system consisting of $\mathrm{He}$ atoms on a graphite surface has attracted considerable attention in recent years. ${ }^{1,2}$ The very earliest theoretical treatments ${ }^{3}$ of this problem made three simplifying assumptions: (a) The atomic motion along the surface is confined to a plane; (b) the periodic component of the surface potential can be neglected; (c) the He-He interaction is the same as that in free space. Comprehensive studies by a variety of experimental techniques (atomic beam ${ }^{4}$ and neutron scattering, ${ }^{5}$ thermodynamic measurements ${ }^{6-8}$ ) have stimulated a succession of calculations which relax these assumptions ${ }^{9-13}$ and a rather consistent overall agreement between the computed and measured results at low coverage has emerged.

One point of disagreement between theory and experiment is the specific heat ${ }^{8}$ in the temperature interval $3<T<5 \mathrm{~K}$. This region is particularly intriguing because band-structure effects are strongly manifest there. ${ }^{10,12}$ Since the calculations based on the atomic scattering data $^{3,4}$ have incorporated these semiempirically, it is somewhat disconcerting to find a non-negligible disagreement.

A consideration omitted from previous analyses is the effect of substrate motion. It is evident that the rigidlattice approximation will fail when the atom is strongly or closely bound or when the substrate is "soft." In fact, the He-graphite binding energy is small, with a well depth $\simeq 15 \mathrm{meV}^{9}$ With respect to softness, there is a lowvelocity branch (transverse acoustic, or TA) of the graphite phonon spectrum ${ }^{14}$ which might couple strongly to the adatom. In this paper we examine coupling to this branch as a possible cause of the discrepancy cited above.

The motion of a particle near a deformable medium is a member of a class of "polaron" problems, in which a single particle interacts with a continuum field. For a liquid-He substrate, the electronic surface polaron ${ }^{15,16}$ and the motion of $\mathrm{H}$ atoms ${ }^{17,18}$ have been studied. However, the graphite case is more complicated because of the extreme elastic anisotropy. The term "surfon" seems appropriate to the atom dressed by surface deformation.

This paper reports results for a somewhat simplified model of the He-graphite surfon. We have treated the static potential as laterally invariant, so that the unperturbed states for motion along the surface are plane waves rather than Bloch waves. Moreover, we have taken the substrate at zero temperature. Nevertheless, we find energy shifts of the right order of magnitude to explain the specific-heat discrepancy, but without correct inclusion of the periodic surface potential we cannot do so.

\section{CALCULATION}

We consider a single adsorbed atom on the planar surface of a homogeneous solid medium which couples to density fluctuations within that medium. Let $W\left(\left|\overrightarrow{\mathrm{r}}-\overrightarrow{\mathrm{r}}^{\prime}\right|\right) \rho\left(\overrightarrow{\mathrm{r}}^{\prime}\right) d^{3} r^{\prime}$ be the potential between the atom at $\vec{r}$ and a volume element $d^{3} r^{\prime}$ of the medium, whose density is $\rho\left(\vec{r}^{\prime}\right)$. The change in total atom-substrate potential due to density fluctuations $\delta \rho\left(\vec{r}^{\prime}\right)$ is

$$
\delta V(\overrightarrow{\mathrm{r}})=\int d^{3} r^{\prime} \delta \rho\left(\overrightarrow{\mathrm{r}}^{\prime}\right) W\left(\left|\overrightarrow{\mathrm{r}}-\overrightarrow{\mathrm{r}}^{\prime}\right|\right) .
$$

For a displacement field $\overrightarrow{\mathrm{u}}\left(\overrightarrow{\mathrm{r}}^{\prime}\right), \delta \rho\left(\overrightarrow{\mathrm{r}}^{\prime}\right)=-\vec{\nabla} \cdot\left[\rho \overrightarrow{\mathrm{u}}\left(\overrightarrow{\mathrm{r}}^{\prime}\right)\right]$, so that ${ }^{19}$

$$
\begin{aligned}
\delta V(\overrightarrow{\mathbf{r}}) & =-\int d^{3} r^{\prime} \vec{\nabla}^{\prime} \cdot\left[\rho \overrightarrow{\mathbf{u}}\left(\overrightarrow{\mathbf{r}}^{\prime}\right)\right] W\left(\left|\overrightarrow{\mathbf{r}}-\overrightarrow{\mathrm{r}}^{\prime}\right|\right) \\
& =\int d^{3} r^{\prime} \rho \overrightarrow{\mathrm{u}} \cdot \nabla^{\prime} W\left(\left|\overrightarrow{\mathrm{r}}-\overrightarrow{\mathrm{r}}^{\prime}\right|\right) .
\end{aligned}
$$

We now write the atom's position as $\vec{r}=(\vec{R}, z)$, i.e., components parallel and perpendicular to the surface, take the substrate to occupy the half-space $z<0$, and define the two-dimensional Fourier transforms

$$
\begin{gathered}
W_{\overrightarrow{\mathrm{K}}}\left(z-z^{\prime}\right)=\int d^{2} R^{\prime} W\left[\left|(0, z)-\left(\overrightarrow{\mathrm{R}}^{\prime}, z^{\prime}\right)\right|\right] e^{-i \overrightarrow{\mathrm{K}} \cdot \overrightarrow{\mathrm{R}}} \\
=\int d^{2} R^{\prime} W\left\{\left[R^{\prime 2}+\left(z-z^{\prime}\right)^{2}\right]^{1 / 2}\right\} e^{-i \overrightarrow{\mathrm{K}} \cdot \overrightarrow{\mathrm{R}}} \\
\overrightarrow{\mathrm{u}}_{\overrightarrow{\mathrm{K}}}\left(z^{\prime}\right)=\int d^{2} R^{\prime} \overrightarrow{\mathrm{u}}\left(\overrightarrow{\mathrm{R}}^{\prime}, z^{\prime}\right) e^{-i \overrightarrow{\mathrm{K}} \cdot \overrightarrow{\mathrm{R}}} \\
\delta V_{\overrightarrow{\mathrm{K}}}(z)=\int d^{2} R \delta V(\overrightarrow{\mathbf{R}}, z) e^{-i \overrightarrow{\mathrm{K}} \cdot \overrightarrow{\mathrm{R}}} \\
=-i \int_{-\infty}^{0} d z^{\prime} \rho \overrightarrow{\mathrm{u}}_{K} \cdot \overrightarrow{\mathrm{K}} W_{K}\left(z-z^{\prime}\right) \\
\quad+\int_{-\infty}^{0} d z^{\prime} \rho u_{K z} \frac{\partial W_{K}\left(z-z^{\prime}\right)}{\partial z^{\prime}}
\end{gathered}
$$

where the last line follows from the convolution theorem.

Displacements of the substrate contribute to the selfenergy of the surfon through the potential $\delta V$. Classically, the adatom carries a stress field with it, induced by its attraction of the substrate; quantum mechanically, the adatom emits and absorbs virtual phonons. When the surfon speed is greater than that of sound in the substrate, the emission of real phonons is kinematically possible. This is analogous to Cerenkov radiation (a sonic boom), and arises naturally in our calculation as an imaginary part of the self-energy.

The unperturbed eigenstates and energies of the adatom are

(c) 1983 The American Physical Society 


$$
\begin{aligned}
& |\overrightarrow{\mathbf{P}}, n\rangle=e^{i \overrightarrow{\mathbf{P}} \cdot \overrightarrow{\mathbf{R}}}|n\rangle, \\
& E_{\overrightarrow{\mathbf{P}}, n}=\hbar^{2} P^{2} / 2 m+E_{n},
\end{aligned}
$$

where $|n\rangle$ is a one-dimensional bound state of energy $E_{n}$ in the static potential $V(z)$, which binds the adatom to the surface, $\overrightarrow{\mathbf{P}}$ is the adatom lateral momentum, and $m$ is the adatom mass. The intermediate states which enter the perturbation calculation are $\left|\overrightarrow{\mathbf{P}}-\overrightarrow{\mathbf{K}}, n^{\prime}, \overrightarrow{\mathbf{k}}\right\rangle$, which consist of a virtual phonon of momentum $\hbar \overrightarrow{\mathrm{k}}=\hbar\left(\overrightarrow{\mathrm{K}}, k_{z}\right)$ and energy $\hbar \omega_{k}$, and the adatom with momentum $\hbar \overrightarrow{\mathrm{P}}-\hbar \overrightarrow{\mathrm{K}}$ in bound state $\left|n^{\prime}\right\rangle$. To second order, the self-energy contribution is (in the limit $\eta \rightarrow 0^{+}$)

$$
\begin{aligned}
\Delta E_{\overrightarrow{\mathrm{P}}, n} & =-\sum_{f} \frac{\left.\langle f|\delta V| i\rangle\right|^{2}}{E_{f}-E_{i}+i \eta} \\
& =-\sum_{n^{\prime}, k} \frac{\left|\left\langle\overrightarrow{\mathrm{P}}-\overrightarrow{\mathrm{K}}, n^{\prime}, \overrightarrow{\mathrm{k}}\left|\delta V_{K}\right| \overrightarrow{\mathrm{P}}, n\right\rangle\right|^{2}}{E_{\overrightarrow{\mathrm{P}}-\overrightarrow{\mathrm{K}}, n^{\prime}}+\hbar \omega_{k}-E_{\overrightarrow{\mathrm{P}}, n}+i \eta} .
\end{aligned}
$$

The phonon spectrum of graphite is characterized by both bulk and Rayleigh (surface) terms, the latter having a displacement field which decays exponentially into the substrate. Each of these modes contributes independently to the self-energy as does each bound state $\left|n^{\prime}\right\rangle$. If we label these terms in (7) $\Delta E_{n}^{B}$ and $\Delta E_{n}^{R}$, and their numerators $\left|M_{n^{\prime} n}^{B}\right|^{2}$ and $\left|M_{n^{\prime} n}^{R}\right|^{2}$, respectively, we can write for the bulk term

$$
\begin{aligned}
\Delta E_{n}^{B}=-\sum_{n^{\prime}} \int \frac{d^{2} K d k_{z}}{(2 \pi)^{3}}\left|M_{n^{\prime} n}^{B}\right|^{2} \\
\times\left[\frac{\hbar^{2} K^{2}}{2 m}-\frac{\hbar^{2} \overrightarrow{\mathbf{P}} \cdot \overrightarrow{\mathrm{K}}}{m}+\hbar \omega_{k}\right. \\
\left.\quad+E_{n^{\prime}}-E_{n}+i \eta\right]^{-1} .
\end{aligned}
$$

By lateral invariance, the matrix element $M_{n^{\prime} n}$ does not depend on the angle between $\overrightarrow{\mathrm{P}}$ and $\overrightarrow{\mathrm{K}}$, so that we can car- ry out the integration over this angle,

$$
\begin{aligned}
\Delta E_{n}^{B}=-\sum_{n^{\prime}} \int \frac{d K d k_{z}}{(2 \pi)^{2}} K\left|M_{n^{\prime} n}^{B}\right|^{2} \\
\times\left[\left(\frac{\hbar^{2} K^{2}}{2 m}+\hbar \omega_{k}+E_{n^{\prime}}-E_{n}\right]^{2}\right. \\
\left.-\left[\frac{\hbar^{2} P K}{m}\right]^{2}\right]^{-1 / 2} .
\end{aligned}
$$

The expression for $\Delta E_{n}^{R}$ is similar, but without the integral $d k_{z} / 2 \pi$. Note that when $P$ is large enough for the energy denominator to be negative, $\Delta E$ becomes imaginary, due to transitions changing either the lateral kinetic energy, the perpendicular state, or both. The decay rate of the surfon is given by $\Gamma_{P_{n}}=2 \operatorname{Im} \Delta E_{P_{n}} / \hbar$.

We now model the polarization and dispersion relation for the important parts of the graphite phonon spectrum. These are low-speed phonons, since the adatom speed at a few degrees Kelvin is only a few hundred meters per second. We assume that the graphite surface is in the basal or cleavage plane, and define an $x$ axis in this plane parallel to the phonon lateral momentum $\overrightarrow{\mathbf{K}}$. Note that polarizations in the $y$ direction decouple completely, as can be seen from (5). Let the phonon displacement field be

$$
\overrightarrow{\mathrm{u}}\left(\overrightarrow{\mathrm{r}}^{\prime}\right)=\operatorname{Re}\left[\left(U, 0, u_{z}\right) \exp \left(i \overrightarrow{\mathrm{K}} \cdot \overrightarrow{\mathrm{R}}^{\prime}+i k_{z} z^{\prime}+i \omega_{k} t\right)\right] .
$$

Then, according to Ref. 20, the secular equation for the dispersion relation is

$$
\left[\begin{array}{ll}
c_{11} K^{2}+c_{44} k_{z}^{2} & \left(c_{44}+c_{13}\right) K k_{z} \\
\left(c_{44}+c_{13}\right) K k_{z} & c_{44} K^{2}+c_{33} k_{z}^{2}
\end{array}\right]\left[\begin{array}{c}
U \\
u_{z}
\end{array}\right]=\rho \omega_{k}^{2}\left[\begin{array}{c}
U \\
u_{z}
\end{array}\right],
$$

where the $c_{i j}$ are the graphite elastic constants, ${ }^{21}$ which are listed in Table I. There are two orthogonal polarizations and two sound speeds. In Fig. 1 we show $\omega_{k} / K$ and the angle $\varphi=\arctan \left(u_{z} / U\right)$ between the polarization vector and the surface for the lowest-speed TA mode. These are

TABLE I. Numerical values of the parameters used.

\begin{tabular}{clc}
\hline \hline Symbol & \multicolumn{1}{c}{ Meaning } & Value \\
\hline$m$ & Adatom bare mass & $4.003 \mathrm{amu}$ \\
$\rho$ & Graphite density & $2.29 \mathrm{~g} \mathrm{~cm}^{-3}$ \\
$c_{0}$ & Minimum sound speed & $142 \mathrm{~ms}^{-1}$ \\
$c_{1}$ & Sound speed change with $K$ & $599 \mathrm{~ms}^{-1} \AA$ \\
$c_{11}$ & Elastic constants of graphite & 106 \\
$c_{44}$ & $\left(10^{11}\right.$ dyn cm $\left.{ }^{-2}\right)$ & 0.40 \\
$c_{13}$ & & 1.50 \\
$c_{33}$ & & 3.65 \\
$\kappa / K$ & Ratio wavelength to decay length & 0.21 \\
& for Rayleigh waves & \\
$E_{0}$ & Energies of perpendicular states & -12.06 \\
$E_{1}$ & (meV) & -6.36 \\
$E_{2}$ & & -2.85 \\
$D$ & Morse potential parameters & $-15.23 \mathrm{meV}$ \\
$\delta$ & & $0.80 \AA$ \\
$z_{m}$ & & $2.70 \AA$ \\
\hline \hline
\end{tabular}




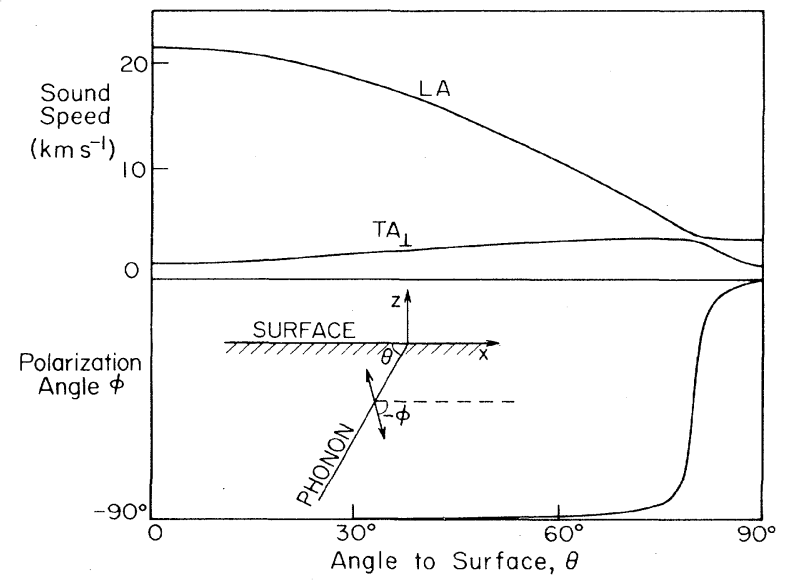

FIG. 1. Speed of longitudinal- (LA) and transverse- (TA) acoustic phonons in graphite, and polarization of the slow TA branch.

functions of the angle $\vartheta=\arctan \left(k_{z} / K\right)$ between the phonon momentum and the surface. The polarization of this mode is approximately normal to the surface for $\vartheta \leq 80^{\circ}$ and parallel for $\vartheta \geq 80^{\circ}$. However, the linear relation between frequency and momentum $\overrightarrow{\mathrm{k}}$ predicted by Eq. (10) does not hold experimentally: The sound speed changes with $\overrightarrow{\mathrm{k}}$ for fixed $\vartheta$. Nicklow et al. ${ }^{14}$ have shown that the TA mode has a minimum sound speed of $\sim 140 \mathrm{~ms}^{-1}$ at $\vartheta=0$, which corresponds to an adatom kinetic energy of $\sim 0.4 \mathrm{meV}$, while the value derived from the elastic constants is $\sim 1300 \mathrm{~ms}^{-1}$, corresponding to $\sim 35 \mathrm{meV}$. Fitting the Nicklow data for $\vartheta=0$, we obtained

$$
\omega_{k}(0)=c_{0} K+c_{1} K^{2},
$$

with $c_{0}, c_{1}$ given in Table I. We assume that all other phonon modes, which have higher speed, are negligible.

To compute the self-energy, we need to know both the phonon dispersion relation and displacement field. Since experimental data are only available for the dispersion relation at $\vartheta=0$, we suitably scale the linear elastic theory to determine the required quantities. Thus we put

$$
\omega_{k}(\vartheta)=\omega_{k}(0)\left[1+\frac{c_{33}}{c_{44}} \tan ^{2} \vartheta\right]^{1 / 2} .
$$

For $\vartheta \geq 80^{\circ}$, the interaction with the adatom is negligible since $u_{z} \approx 0$ and $K \ll k_{z}$.

To evaluate the required matrix elements, we assume that the adatom is bound to the surface by a Morse potential

$$
V(z)=D\left(e^{2\left(z_{m}-z\right) / \delta}-2 e^{\left(z_{m}-z\right) / \delta}\right),
$$

which corresponds to ${ }^{19}$

$$
W(\overrightarrow{\mathbf{r}})=\frac{D}{\pi \rho \delta^{2} x}\left(2 e^{-2\left(x-z_{m}\right) / \delta}-e^{-\left(x-z_{m}\right) / \delta}\right),
$$

where $\rho$ is the substrate density. By fitting the observed bound-state energies of the perpendicular states ${ }^{4}$ to the Morse energy levels,

$$
E_{n}=D+\hbar \omega\left(n+\frac{1}{2}\right)-\hbar^{2} \omega^{2}\left(n+\frac{1}{2}\right)^{2} / 4 D,
$$

where $\omega^{2}=2 D / m \delta^{2}$, we obtain the parameters shown in Table I.

The phonon normalization is such that the total energy of the displacement field is $\hbar \omega_{k}$ per unit volume (area) for the bulk (Rayleigh) waves. By the virial theorem, this energy is equipartitioned between kinetic and potential energy. Equation (5) therefore gives

$$
\begin{aligned}
M_{n^{\prime} n}^{B}=\frac{4 D}{\delta^{2}}\left[\frac{\hbar}{2 \rho \omega_{k}}\right]^{1 / 2} & {\left[e^{2 z_{m} / \delta} \frac{\left\langle n^{\prime}\left|e^{-K_{4} z}\right| n\right\rangle}{K_{4}+i k_{z}}\right.} \\
& \left.-\frac{1}{2} e^{z_{m} / \delta} \frac{\left\langle n^{\prime}\left|e^{-K_{1} z}\right| n\right\rangle}{K_{1}+i k_{z}}\right],
\end{aligned}
$$

where $K_{4}^{2}=K^{2}+4 \delta^{-2}$ and $K_{1}^{2}=K^{2}+\delta^{-2}$. The expression for $M_{n^{\prime} n}^{R}$ (Rayleigh waves) is similar, except that an extra factor $\kappa^{1 / 2}$ appears, where the wave amplitude at depth $-z^{\prime}$ is proportional to $e^{\kappa z^{\prime}}$, and also $i k_{z}$ is replaced by $\kappa$. Reference 21 gives $\kappa \approx 0.21 \mathrm{~K}$.

The matrix elements of an exponential between Morse states are easily evaluated. We calculated the integral for $\Delta E$ by first changing variables from $K, k_{z}$ to $e^{-K \delta}, \vartheta$ $\left[=\arctan \left(k_{z} / K\right)\right]$, then integrating in $0<-e^{-K \delta}<1$, and for the bulk-wave part also in $0 \leq \vartheta \leq 80^{\circ}$. We used a double Simpson's rule with a large mesh of $500 \times 500$ points, since there is, in general, a line of inverse square-root singularity in the integrand. Even with this large mesh, the results showed fluctuations, which we have checked to be mesh dependent and removed. In Fig. 2 we show for adatom kinetic energy $\hbar^{2} P^{2} / 2 m$ between 0 and $10 \mathrm{meV}$ the contributions to $-\Delta E_{P n}$ and $\Gamma_{P n}$ from a variety of intermediate states $\left|n^{\prime}\right\rangle$, with the notation $n \rightarrow n^{\prime}$. These curves enable us to calculate the effective masses $m_{n}^{*}$ of the adatom, given by

$$
\frac{m}{m_{n}^{*}}-1=\left(\frac{d \Delta E_{P n}}{d E}\right)_{E=0},
$$

which are shown in Table II with the same notation.

It is also necessary to show that the continuum approximation for the substrate is valid; i.e., that the integrand in Eq. (9) is negligible outside the Brillouin zone. This zone is

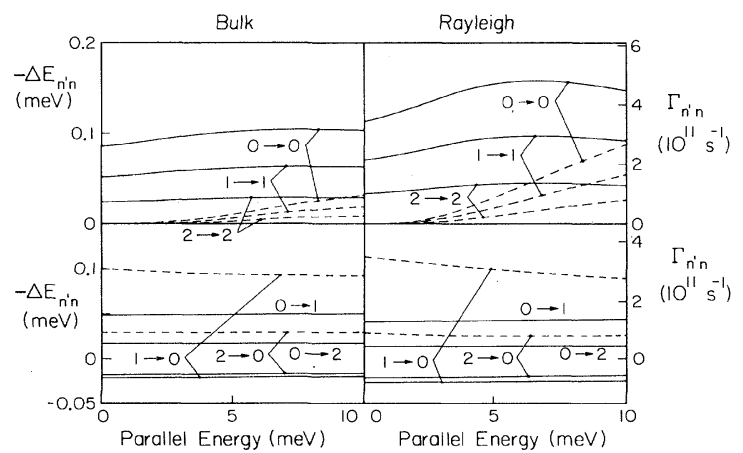

FIG. 2. Contributions to the self-energy of the surfon, as a function of parallel kinetic energy. Left panels are bulk-wave part, right panels are Rayleigh-wave part. Solid lines are real energy; dashed lines are imaginary energy, corresponding to the probability rate on the right-hand scale. 
TABLE II. Effective mass increases due to polaron effects for ${ }^{4} \mathrm{He}$ on graphite for various initial and intermediate perpendicular state combinations.

\begin{tabular}{lccc}
\hline \hline States & Bulk & Rayleigh & Sum \\
\hline $0 \rightarrow 0$ & $0.25 \%$ & $0.59 \%$ & \\
$0 \rightarrow 1$ & $0.02 \%$ & $0.03 \%$ & \\
$0 \rightarrow 2$ & $0.00 \%$ & $0.01 \%$ & \\
& & & $0.90 \%$ \\
$1 \rightarrow 0$ & $0.01 \%$ & $0.03 \%$ & \\
$1 \rightarrow 1$ & $0.15 \%$ & $0.36 \%$ & $0.55 \%$ \\
& & & \\
$2 \rightarrow 0$ & $0.03 \%$ & $0.05 \%$ & $0.32 \%$ \\
\hline \hline
\end{tabular}

of mean radius $1.51 \AA$ parallel to the basal plane, and 0.94 $\AA$ perpendicular to it. The integral over the region outside the zone for all cases shown in Fig. 2 was less than $1 \%$ of the total.

\section{DISCUSSION}

Our calculation shows that Rayleigh-wave contributions to the self-energy and lifetime of the surfon are comparable to the bulk-wave contributions, reemphasizing their importance in surface dynamics. ${ }^{22}$ The values of $\Delta E$ are small, but not negligible. For example, the ground-state shift is $-0.3 \mathrm{meV}$, about $2 \%$ of the measured groundstate energy, ${ }^{7}-12.27 \pm 0.17 \mathrm{meV}$. This shift does not invalidate the agreement with the band-structure result, ${ }^{11}$ $-12.22 \mathrm{meV}$, because only phonon-renormalized energies are measured in experiments. The scattering data used to calculate the adatom band structure were taken at $T \approx 100$ $\mathrm{K} \approx 10 \mathrm{meV}$ and extrapolated to zero temperature, and it is the essential independence of our results to parallel energy that explains the agreement. Furthermore, a thermal phonon at $100 \mathrm{~K}$ has a wave number of $\kappa \approx 0.1 \AA^{-1}$, and this range of $k$ makes a small contribution to the integral in Eq. (9), so we can conclude that our $T=0$ analysis is sufficient for characterizing the single-particle ground state.

Since the energy shift $\Delta E$ is almost independent of parallel energy, the specific heat of a helium film is unchanged by inclusion of phonons. Thus the discrepancy cited in the Introduction remains unexplained, although the inclusion of the periodic part of the surface potential may provide an eventual resolution. In fact, our selfenergy corrections are large enough compared to the thermal energy in the specific-heat experiments that one might be optimistic. A substitution of Bloch waves for plane waves in Eq. (6) is required, and we hope to use this approach in future work.

The size of the calculated lifetimes $\Gamma^{-1} \approx 10^{-11} \mathrm{~s}$ is to be expected from the size of the self-energy. The decrease of $\Gamma$ with increasing $n$ arises from the weaker coupling of the phonons at larger distances. Such behavior has been seen in "selective evaporation" resonance patterns observed in scattering of $\mathrm{He}$ from $\mathrm{LiF}^{23}$ The following values of $\Gamma_{n}$ were obtained: $\Gamma_{0}=1.4, \Gamma_{1}=0.9, \Gamma_{2}=0.6$, in units of $10^{11} \mathrm{~s}^{-1}$. Roughly comparable values are seen in Fig. 2 for the relevant value $10 \mathrm{meV}$ of parallel energy. Naturally, the different binding energies and elastic properties obviate a detailed comparison. Note that at this parallel energy, the mean free path is $20-30 \AA$.

In summary, there is some prospect for a significant effect on the specific heat, with incorporation of the periodic potential. The scattering rate values are in agreement with expectation, and a measurement would provide a useful confirmation.

\section{ACKNOWLEDGMENTS}

We are grateful to Flavio Toigo and David Goodstein for useful discussions. This work was supported by National Science Foundation Grants Nos. PHY-79-23638, PHY-82-07332, DMR-81-13262, and U. S. Department of Energy Contract No. DE-AM03-76SF00767.
1J. G. Dash and M. Schick, in Physics of Liquid and Solid Helium, edited by K. H. Bennemann and J. B. Ketterson (Wiley, New York, 1979).

${ }^{2}$ M. W. Cole, D. R. Frankl, and D. L. Goodstein, Rev. Mod. Phys. 53, 199 (1981).

${ }^{3}$ R. L. Siddon and M. Schick, Phys. Rev. A 9, 907 (1974); 9 , 1753 (1974).

${ }^{4}$ G. Boato, P. Cantini, C. Guidi, R. Tatarek, and G. P. Felcher, Phys. Rev. B 20, 3957 (1979); G. Derry, D. Wesner, W. E. Carlos, and D. R. Frankl, Surf. Sci. 87, 629 (1979).

${ }^{5}$ K. Carneiro, L. Passell, W. Thomlinson, and H. Taub, Phys. Rev. B 24, 1170 (1981).

${ }^{6}$ M. Bretz, J. G. Dash, D. C. Hickernell, E. O. McLean, and O. E. Vilches, Phys. Rev. A $\underline{8}, 1589$ (1973).

${ }^{7}$ R. L. Elgin and D. L. Goodstein, Phys. Rev. A 9, 2657 (1974); R. L. Elgin, J. M. Greif, and D. L. Goodstein, Phys. Rev. Lett. 41, 1723 (1978).

${ }^{8}$ A. F. Silva-Moreira, J. Codona, and D. L. Goodstein, Phys.
Lett. 76A, 324 (1980).

${ }^{9}$ W. E. Carlos and M. W. Cole, Surf. Sci. 91, 339 (1980).

${ }^{10}$ W. E. Carlos and M. W. Cole, Phys. Rev. B 21, 3713 (1980).

${ }^{11}$ M. W. Cole and F. Toigo, Phys. Rev. B 23, 3914 (1981).

${ }^{12}$ L. W. Bruch, Phys. Rev. B 23, 6801 (1981); Z.-C. Guo and L. W. Bruch (unpublished).

${ }^{13}$ G. Vidali and M. W. Cole, Phys. Rev. B $\underline{22}, 4661$ (1980); 23, 5649 (1981).

${ }^{14}$ R. Nicklow, N. Wakabayashi, and H. G. Smith, Phys. Rev. B 12, 4951 (1972).

${ }^{15}$ T. S. Rahman and D. L. Mills, Phys. Rev. B 21; 1432 (1980).

${ }^{16}$ S. A. Jackson and P. L. Platzman, Phys. Rev. B 24, 499 (1981).

${ }^{17}$ P. L. Platzman (private communication).

${ }^{18}$ R. Guyer, Phys. Rev. B 23, 4570 (1982).

${ }^{19}$ Note that the long-wavelength limit of Eq. (2) is $-\overrightarrow{\mathbf{U}}(\overrightarrow{\mathbf{R}}, 0) \cdot \vec{\nabla} \boldsymbol{V}(\overrightarrow{\mathbf{r}})$. See M. W. Cole and F. Toigo, Surf. Sci. 119 , L346 (1982) for this expansion and the case of a discrete lattice. 
${ }^{20}$ K. F. Graff, Wave Motion in Elastic Solids (Ohio State University Press, Columbus, 1975).

${ }^{21}$ E. de Rouffignac, G. P. Alldredge, and F. W. de Wette, Phys. Rev. B 23, 4208 (1981).
22J. E. Black, B. Laks, and D. L. Mills, Phys. Rev. B 22, 1818 (1980).

${ }^{23}$ G. Brusdeylins, R. B. Doak, and J. P. Toennies, J. Chem. Phys. 75, 1784 (1981). 